\title{
When an old star smolders
}

\section{On the detection of hydrocarbon emission from S-type AGB stars}

\author{
K. Smolders ${ }^{1, \star}$, B. Acke ${ }^{1, \star \star}$, T. Verhoelst ${ }^{1, \star \star}$, J. A. D. L. Blommaert ${ }^{1}$, L. Decin ${ }^{1,4, \star \star}$, S. Hony $^{2}$, \\ G. C. Sloan ${ }^{3}$, P. Neyskens ${ }^{5, \star \star \star}$, S. Van Eck ${ }^{5}$, A. A. Zijlstra ${ }^{6}$, and H. Van Winckel ${ }^{1}$ \\ 1 Instituut voor Sterrenkunde (IvS), Katholieke Universiteit Leuven, Celestijnenlaan 200 D, 3001 Leuven, Belgium \\ e-mail: kristof.smolders@ster.kuleuven.be \\ 2 Service d'Astrophysique, CEA Saclay, Bât.709, Orme des Merisiers, 91191 Gif-sur-Yvette, France \\ 3 Cornell University, Astronomy Department, Ithaca, NY 14853, USA \\ 4 Universiteit van Amsterdam, Sterrenkundig Instituut "Anton Pannekoek", Kruislaan 403, 1098 SJ Amsterdam, The Netherlands \\ 5 Institut d'Astronomie et d'Astrophysique (IAA), Université Libre de Bruxelles, C.P. 226, Boulevard du Triomphe, 1050 Bruxelles, \\ Belgium \\ 6 Jodrell Bank Centre for Astrophysics, Alan Turing Building, University of Manchester, Oxford Road, Manchester, M13 9PL, UK
}

Received 12 February 2010 / Accepted 9 April 2010

\section{ABSTRACT}

\begin{abstract}
Polycyclic aromatic hydrocarbons (PAHs) produce characteristic infrared emission bands that have been observed in a wide range of astrophysical environments, where carbonaceous material is subjected to ultraviolet (UV) radiation. Although PAHs are expected to form in carbon-rich AGB stars, they have up to now only been observed in binary systems where a hot companion provides a hard radiation field. In this letter, we present low-resolution infrared spectra of four S-type AGB stars, selected from a sample of 90 S-type AGB stars observed with the infrared spectrograph aboard the Spitzer satellite. The spectra of these four stars show the typical infrared features of PAH molecules. We confirm the correlation between the temperature of the central star and the centroid wavelength of the $7.9 \mu \mathrm{m}$ feature, present in a wide variety of stars spanning a temperature range from 3000 to $12000 \mathrm{~K}$. Three of four sources presented in this paper extend this relation towards lower temperatures. We argue that the mixture of hydrocarbons we see in these S-stars has a rich aliphatic component. The fourth star, BZ CMa, deviates from this correlation. Based on the similarity with the evolved binary TU Tau, we predict that BZ CMa has a hot companion as well.
\end{abstract}

Key words. stars: AGB and post-AGB - stars: mass-loss - stars: winds, outflows, ISM: molecules

\section{Introduction}

Polycyclic aromatic hydrocarbon (PAH) molecules are large molecules containing carbon and hydrogen atoms. They carry typical infrared (IR) emission features that have been observed in many astrophysical environments (Tielens 2008). Since these features are generally attributed to the IR fluorescence of ultraviolet-pumped molecules, we expect PAH features to arise from regions where carbon-rich material is exposed to ultraviolet (UV) radiation (Leger \& Puget 1984; Cohen et al. 1985; Puget \& Leger 1989; Allamandola et al. 1989). PAHs play a major role in photoelectric heating processes and the ionization balance of the interstellar material (Lepp \& Dalgarno 1988).

Although interstellar PAH molecules are thought to originate in the winds of carbon-rich asymptotic giant branch (AGB) stars, there is little observational support for this idea. In a complete ISO/SWS survey of 50 carbon-rich AGB stars, Boersma et al. (2006) detected PAH emission in only one source, TU Tau, a carbon-rich AGB star with a hot A2 companion providing UV radiation. Recently, Sloan et al. (2007) has detected PAH emission in an R-type carbon-rich giant with a circumstellar disk, which is the likely region where the PAH emission originates. This lack of PAH detections in carbon-rich AGB stars is most likely the result of the high optical opacities of carbon-rich

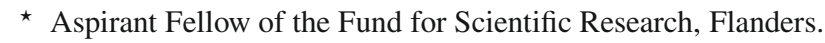

$\star \star$ Postdoctoral Fellows of the Fund for Scientific Research, Flanders.

$\star \star \star$ Fellowship “boursier F.R.I.A", Belgium. dust: when formed, the PAH molecules are shielded from optical and ultraviolet photons by the opaque carbonaceous circumstellar material (Jäger et al. 1998).

S-type stars are objects on the ascent of the AGB. They pass through the phase where the photosphere turns from oxygenrich to carbon-rich $(\mathrm{C} / \mathrm{O} \approx 1)$. Because S-type stars are not yet forming carbon-rich dust, they have less opaque circumstellar shells than their carbon-rich successors (Jäger et al. 2003). Nevertheless, due to shock-induced non-equilibrium chemistry effects, S-type stars could display carbon-rich molecules (Cherchneff 2006). Recent studies of ISO/SWS spectra of S-type AGB stars indeed show the presence of molecules like HCN and $\mathrm{C}_{2} \mathrm{H}_{2}$ (Yang et al. 2007; Hony et al. 2009).

We have studied the data obtained with the Spitzer Infrared Spectrograph (IRS, Houck et al. 2004) of a sample of 90 intrinsic $^{1}$ S-type stars (Program ID 30737, P.I. S. Hony). A description of the sample can be found in Cami et al. (2009). They present the detection of SiS absorption bands, in a large subset of the sample. In this paper, we present the detection of PAH emission around four cool S-type AGB stars. Three of the four objects extend the known correlation between the centroid wavelength of the $7.9 \mu \mathrm{m}$ PAH feature and the stellar effective temperature to lower temperatures and more redshifted features. We argue that the hydrocarbon molecules have a high aliphatic/aromatic ratio.

\footnotetext{
1 Intrinsic S-type stars are not enriched in C or s-process elements by accretion from an evolved companion, but through internal nucleosynthesis.
} 
Table 1. Anchor points of the continuum splines.

\begin{tabular}{ccc}
\hline \hline Feature & \multicolumn{2}{c}{ Anchor Points } \\
& Class B/C sources & Class A sources \\
$\mu \mathrm{m}$ & $\mu \mathrm{m}$ & $\mu \mathrm{m}$ \\
\hline 6.2 & $5.7,5.9,6.1,6.5,6.7,6.9$ & idem \\
7.9 & $7.0,7.2,7.4,9,9.2,9.4$ & $6.6,6.8,7.0,9.0,9.2,9.4$ \\
11.2 & $10.3,10.5,10.7,11.8,12.0,12.2$ & idem \\
\hline
\end{tabular}

Since AGB stars are important producers of the dust in the interstellar medium, we might be looking at the hydrocarbon mixture, as formed in the current day wind.

\section{Observations and data reduction}

The Spitzer IRS spectra were obtained in the low-resolution staring mode, which places the star in two nod positions. This is first done in the short-low (SL) apertures, and then in two longlow (LL) apertures. The resulting spectrum covers the 5 to $37 \mu \mathrm{m}$ region with a typical resolution of $R \sim 100$.

For the data reduction we used the FEPS pipeline, developed for the Spitzer legacy program "Formation and evolution of planetary systems". A detailed description can be found in Hines et al. (2005). For the extraction we used the intermediate droop data products as delivered by the SSC, together with the SMART reduction package tools described in detail in Higdon et al. (2004). The spectra are background-corrected by subtracting the images of the two slit positions for each module and order. The IRS spectrum of all stars is de-reddened using the extinction maps from Schlegel et al. (1998) and the IR extinction law of Chiar \& Tielens (2006).

\section{The observed emission features}

The PAH molecules exhibit very characteristic emission features in the 5-14 $\mu \mathrm{m}$ region, the most prominent at $6.2,7.9,8.6$, and $11.2 \mu \mathrm{m}$. These features arise from the bending and stretching of the carbon and hydrogen bonds in the large molecules.

\subsection{Extraction of the PAH features}

The S-type stars in the sample show a wide variety of infrared spectra, containing photospheric absorption bands of e.g. CO, $\mathrm{H}_{2} \mathrm{O}, \mathrm{SiO}$, and $\mathrm{SiS}$. The absorption bands in combination with the weak emission features make it difficult to estimate an accurate continuum. The method of extracting possible emission features combines the use of cubic splines for the 6.2 and $11.2 \mu \mathrm{m}$ features and comparison stars for the 7.9-8.6 $\mu \mathrm{m}$ complex.

For the regions around 6.2 and $11.2 \mu \mathrm{m}$, the continuum flux was estimated by fitting a cubic spline (with smoothness zero) through anchor points at different positions next to the features (see Table 1). By applying random variations of the spectra within the calculated error bars and shifting the anchor points 2 spectral points outwards, we obtained a large set of continua. The spread on these continua was used to estimate the error introduced by fitting a spline to the data.

Strong water vapor absorption can mimic the presence of a $6.2 \mu \mathrm{m}$ emission feature (see for example Fig. A.23 in Cami 2002). However, the water vapor bands can be easily recognized by comparing the full spectrum with synthetic spectra, and are generally weak in S-type stars. We excluded five stars in which the detection of a $6.2 \mu \mathrm{m}$ feature was due to this effect.

BZ CMa is the only star in our sample of S-type stars with possible $\mathrm{PAH}$ emission, which does not show the $\mathrm{SiO}$ absorption band in the region containing the 7.9 and $8.6 \mu \mathrm{m} \mathrm{PAH}$

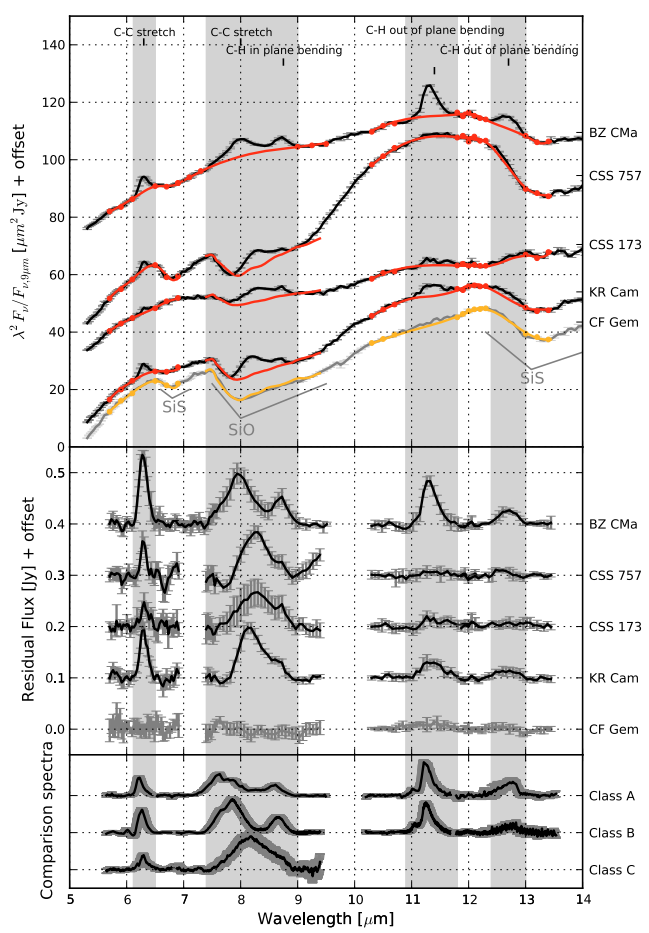

Fig. 1. Top panel: the infrared spectra of the 4 stars (black) and the continuum estimates (red) with the characteristic infrared emission features from PAH molecules, shown in Rayleigh units. As a reference, we show the spectrum of CF Gem, a naked photosphere, with estimated continuum. The spectrum of CSS 757 shows weak PAH emission, as well as a dust emission feature at $11 \mu \mathrm{m}$. Middle panel: the residual flux. Bottom panel: Rescaled ISO-SWS spectra of prototypes of the different PAH classes: NGC 1333-3 (Class A), HD 44179 (Class B) and CRL 2688 (Class C) (Peeters et al. 2002; van Diedenhoven et al. 2004).

emission features. For this star, we used a spline at the anchor points mentioned in Table 1 to estimate the continuum. The other stars contain the $\mathrm{SiO}$ band head, which varies in depth throughout the sample of S-type stars. Because this absorption band is asymmetrical, a spline is not a good estimate of the photospheric continuum. Instead, we used the 40 out of 90 sample stars without dust emission (naked photospheres) as template stars, fitted in the regions $7.4-7.9 \mu \mathrm{m}$ and $9-9.4 \mu \mathrm{m}$ where the contribution of the PAH emission is negligible. We used the spread on the 5 best-fitting template stars as a conservative estimate of the error caused by fitting these spectra.

We calculated the integrated flux of the three continuumsubtracted features. When all features had a line flux exceeding the 3-sigma level, we considered this a positive detection of $\mathrm{PAH}$ emission. In Table 2 we present the 4 stars with a positive detection of PAH emission. Figure 1 shows the spectra, the estimated continua and the residual fluxes of these stars. We include the spectrum of CF Gem, a star without PAH emission, for comparison and to indicate the quality of the continuum estimates.

The detected PAH features in the infrared spectra can have an interstellar origin. To exclude this possibility, the background spectrum in the off-source nod (i.e. in the other order) was extracted. Since no significant features were found in the background spectra, we conclude that the PAH emission comes from the immediate environment of the star. Moreover, the feature profiles are very different from those observed in interstellar sources. From here on we focus only on these 4 stars with a positive detection of PAHs: BZ CMa, KR Cam, CSS 173, and CSS 757. 
K. Smolders et al.: When an old star smolders

Table 2. The 4 S-type stars with significant emission features at $6.2,7.9$, and $11.2 \mu \mathrm{m}$.

\begin{tabular}{|c|c|c|c|c|c|c|c|c|}
\hline \multicolumn{3}{|c|}{ Stellar parameters } & \multicolumn{2}{|l|}{$6.2 \mu \mathrm{m}$} & \multicolumn{3}{|c|}{$7.9 \mu \mathrm{m}$} & \multirow{2}{*}{$\begin{array}{c}11.2 \mu \mathrm{m} \\
\text { Flux }^{b} \\
\mathrm{erg} \mathrm{cm}^{-2} \mathrm{~s}^{-1}\end{array}$} \\
\hline Name & $\begin{array}{c}T_{\mathrm{eff}^{a}}{ }^{\mathrm{K}} \\
\end{array}$ & $\mathrm{C} / \mathrm{O}^{a}$ & $\begin{array}{c}\text { Flux }^{b} \\
\operatorname{erg~cm}^{-2} \mathrm{~s}^{-1}\end{array}$ & Class $^{c}$ & $\begin{array}{c}\lambda_{\text {peak }} \\
\mu \mathrm{m}\end{array}$ & $\begin{array}{l}\lambda_{\text {cen }} \\
\mu \mathrm{m}\end{array}$ & $\begin{array}{c}\text { Flux }^{b} \\
\operatorname{erg~cm}^{-2} \mathrm{~s}^{-1}\end{array}$ & \\
\hline CSS 173 & $3600 \pm 150$ & $0.97 \pm 0.01$ & $3.0 \pm 0.7 \times 10^{-13}$ & $\mathrm{C}$ & $8.25 \pm 0.05$ & $8.38 \pm 0.10$ & $1.6 \pm 0.2 \times 10^{-12}$ & $1.1 \pm 0.2 \times 10^{-13}$ \\
\hline KR Cam & $3400 \pm 150$ & $0.97 \pm 0.01$ & $6.2 \pm 0.6 \times 10^{-13}$ & $\mathrm{C}$ & $8.16 \pm 0.05$ & $8.31 \pm 0.06$ & $1.5 \pm 0.1 \times 10^{-12}$ & $1.3 \pm 0.2 \times 10^{-13}$ \\
\hline $\mathrm{BZ} \mathrm{CMa}$ & $3400 \pm 150$ & $0.98 \pm 0.01$ & $20 \pm 2 \times 10^{-13}$ & B & $7.97 \pm 0.04$ & $8.02 \pm 0.04$ & $3.4 \pm 0.2 \times 10^{-12}$ & $9.8 \pm 0.5 \times 10^{-13}$ \\
\hline CSS 757 & $3400 \pm 150$ & $0.97 \pm 0.01$ & $5.6 \pm 0.7 \times 10^{-13}$ & $\mathrm{C}$ & $8.27 \pm 0.05$ & $8.47 \pm 0.06$ & $2.2 \pm 0.2 \times 10^{-12}$ & $1.1 \pm 0.3 \times 10^{-13}$ \\
\hline
\end{tabular}

Notes. ${ }^{(a)}$ Derived from the comparison of low-resolution spectroscopy with a dedicated grid of MARCs model atmospheres for S-type stars (Plez et al. in preparation, Gustafsson et al. 2008). The low-resolution spectroscopy was obtained for the complete sample of S-type stars, with the William Hershel Telescope on La Palma for the sources in the northern sky (range: $4100 \AA-8300 \AA$; resolution: $2 \AA$ ) and with the $1.9 \mathrm{~m}$ telescope at the South African Astronomical Observatory for the southern targets (range: $4900 \AA-7800 \AA$; resolution: $4 \AA$ ). The entire dataset will be presented in a forthcoming paper. ${ }^{(b)}$ Integrated line flux of the residual flux in the interval [6.12,6.5] $\mu \mathrm{m},[7.4,9.0] \mu \mathrm{m}$, or $[10.9,11.8] \mu \mathrm{m}$. (c) According to Peeters et al. (2002).

\subsection{Classification}

Peeters et al. (2002) shows that the C-C stretch modes at 6.2 and $7.9 \mu \mathrm{m}$ display a wide spread in peak position. They classify objects in three classes from A (bluest features) over B to C (reddest features). Because of the low spectral resolution and signalto-noise of our data, we focused on the broad feature at $7.9 \mu \mathrm{m}$. For Class $\mathrm{C}$ the peak of this feature is shifted to $8.2 \mu \mathrm{m}$, marking a clear difference with the $7.9 \mu \mathrm{m}$ feature of Class B PAHs and the $7.6 \mu \mathrm{m}$ feature of Class A PAHs. In Table 2, we show the peak wavelengths and the classes we derived for all stars. In Fig. 1 the residual spectra of the S-type stars with PAH features, i.e. after continuum subtraction, are compared with PAH features from each of these 3 classes.

Based on the peak wavelength, BZ CMa is the only star that shows the Class B PAHs, generally found in PNe, Herbig Ae/Be stars, and in some red supergiants (Sylvester et al. 1998; Tielens 2008; Verhoelst et al. 2009). The three other stars are members of the small group of known Class $\mathrm{C}$ sources, together with a few post-AGB stars (Peeters et al. 2002; Kraemer et al. 2006; Gielen et al. 2009), the Herbig Ae/Be source HD 135344B (Sloan et al. 2005), the intermediate-mass T Tauri star SU Aur (Furlan et al. 2006), the carbon-rich red giant HD 100764 (Sloan et al. 2007), and the oxygen-rich K giant HD 233517 (Jura et al. 2006).

\section{Correlation between $T_{\text {eff }}$ and feature redshift}

The centroid wavelength $\left(\lambda_{\text {cen }}\right)$ is the continuum-subtracted, flux-weighted barycenter of the feature. Recently, it has been claimed that the centroid wavelength of the 6.2,7.9, and $11.2 \mu \mathrm{m}$ features are shifted to longer wavelengths in targets with a lower effective stellar temperature (Sloan et al. 2007; Keller et al. 2008; Boersma et al. 2008).

\subsection{Extending the correlation towards cooler stars}

In Fig. 2 we show the centroid wavelength of the $7.9 \mu \mathrm{m}$ feature for all stars in our sample, supplemented by the centroid wavelengths of a comparison sample consisting of all Class C sources with observed $7.9 \mu \mathrm{m}$ features (Kraemer et al. 2006; Furlan et al. 2006; Jura et al. 2006; Sloan et al. 2007), the carbonrich AGB star TU Tau (Boersma et al. 2006), the Herbig Ae stars without silicate emission from Keller et al. (2008), and all PAH sources in Peeters et al. (2002) with stellar temperatures below $20000 \mathrm{~K}$. The continuum flux was estimated by fitting a cubic spline through the anchor points given in Table 1, as described in Sect. 3.1. To calculate the centroid wavelengths, we fit a line to the left and right of the $7.9 \mu \mathrm{m}$ feature and removed the $8.6 \mu \mathrm{m}$

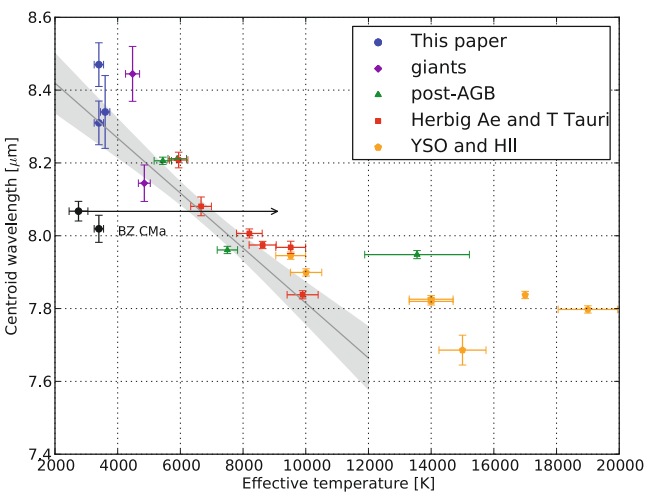

Fig. 2. The centroid wavelength of the $7.9 \mu \mathrm{m}$ feature against the stellar effective temperature. BZ CMa and TU Tau are shown as black circles. The arrow indicates the shift of TU Tau to the temperature of the A2 companion. The gray line and area represent the correlation, quantified in Eq. (1) with the corresponding $99 \%$ confidence interval.

feature. Redefining the centroid wavelength for all sources, using the same methods as for the S-type stars, allows for a quantative description of the correlation.

Our sample confirms the strong correlation between the effective temperature of the central star and the centroid position of the $7.9 \mu \mathrm{m}$ feature. Moreover, the PAH features in the cool S-type stars are redshifted the most. The correlation can be approximated by a linear relationship for all temperatures below $12000 \mathrm{~K}$. A linear regression analysis results in

$$
\lambda_{\text {cen }}[\mu \mathrm{m}]=(8.05 \pm 0.01)-(7.54 \pm 0.60) \times\left(T_{\text {eff }}\left[10^{5} \mathrm{~K}\right]-0.077\right)(1)
$$

with a Pearson correlation coefficient of $R=-0.90$ and a twotailed probability much lower than 0.1 percent, indicating a very strong correlation. All sources with higher temperatures have Peeters Class A features $\left(\lambda_{\text {cen }}=7.84 \pm 0.06 \mu \mathrm{m}\right)$. A similar correlation is present for the 6.2 and $11.2 \mu \mathrm{m}$ features, albeit to a lesser degree.

Two stars that do not follow this linear trend are BZ CMa and TU Tau. The latter, however, is a known spectroscopic binary with a hot A2-type companion $\left(T_{\mathrm{eff}} \approx 9400 \mathrm{~K}\right)$ (Richer 1972). If we use this temperature instead of the effective temperature of the carbon star $(2750 \mathrm{~K})$, this star falls within the scatter on the correlation. For BZ CMa we use Eq. (1) to predict the presence of a hot A7-type companion with a temperature of approximately $8100 \mathrm{~K}$. Additional data is required to check this prediction.

For the three other stars there is no indication of a hot companion. The detection of PAH features in the IR spectra of these 
stars means that PAHs can be sufficiently excited by stars with low effective temperatures, hence soft radiation fields.

\subsection{Aliphatic and aromatic material}

Sloan et al. (2007) propose an explanation for this correlation, based on qualitatively similar shifts in laboratory measurements of PAHs. Class C PAHs are not subjected to strong UV radiation fields and thus are relatively unaltered mixes of aliphatic and aromatic hydrocarbons. Class A and B PAHs have been processed by the hard radiation of the central stars, suppressing the aliphatic component (e.g. Goto et al. 2002, 2003, 2007). This agrees with the results of Pino et al. (2008), who show that Class C sources, with a redshifted $6.2 \mu \mathrm{m}$ feature, have a strong a strong aliphatic emission feature at $3.4 \mu \mathrm{m}$.

The three targets following the correlation at very low temperatures can be classified as extreme Class C sources, with very broad, red emission features. CSS 757, KR Cam and CSS 173, are thus the objects with the highest aliphatic/aromatic content ratio observed in space to date, exhibiting the composition as condensed in their winds.

\section{Discussion}

Merely that we can detect hydrocarbon features around cool stars without a hot companion is remarkable. These detections push the temperature of the radiation field exciting PAHs to even lower values than before. Since these stars do not have strong UV radiation fields, the hydrocarbon mixture must be pumped by optical photons. The theoretical model presented in Li \& Draine (2002) predicts that optical pumping is possible for ionized PAHs and for large PAH molecules with more than 100 carbon atoms. Later refinements of this PAH model, based on laboratory measurements, show that even near-infrared photons can excite ionized PAH molecules (Mattioda et al. 2005a,b). The detection of hydrocarbon emission around the S-type stars implies that the molecules are either ionized or very large, or, alternatively, that they can be excited by optical photons due to the aliphatic component.

The detection of hydrocarbon emission in combination with the $\mathrm{SiO}$ absorption band in the stellar photosphere points to mixed chemistry. This is unexpected if the ongoing chemistry in the circumstellar environment of S-type stars is in thermal equilibrium (Ferrarotti \& Gail 2002). Because the presence of $\mathrm{SiO}$ in the photosphere points to an oxygen-dominated chemistry, the majority of $\mathrm{C}$ atoms are bound in $\mathrm{CO}$ molecules. However, in Cherchneff (2006), the nonequilibrium chemistry in the inner wind of AGB stars is studied, and the results differ strongly from those found by Ferrarotti \& Gail (2002). For stars with an equal amount of carbon and oxygen $(\mathrm{C} / \mathrm{O}=1)$ nonequilibrium, shockdriven chemistry predicts $\mathrm{C}_{2} \mathrm{H}_{2}$ molecules in the outer regions of the stellar outflow. These carbon-rich molecules are the building blocks of PAHs (Cherchneff et al. 1992; Cau 2002). This is consistent with all $\mathrm{C} / \mathrm{O}$ ratios found for these stars being very close to unity (see Table 2). When compared to our entire sample of $90 \mathrm{~S}$-type stars, the $\mathrm{C} / \mathrm{O}$ ratios of these four stars are in the top $10 \%$, with the others being less carbon-rich.

\section{Conclusions}

In this paper we present a positive detection and identification of PAH emission in 4 S-type AGB stars. In this small sample we see a clear difference between the strong PAH emission in BZ CMa, which can be classified as Peeters Class B, and the much weaker $\mathrm{PAH}$ features seen in the other sources of Class $\mathrm{C}$. We predict that BZ CMa is a binary system with a hot, late-A-type companion.

Our data are consistent with the strong correlation found between the centroid wavelength of the $7.9 \mu \mathrm{m}$ feature and the temperature of the central star. They extend this correlation towards lower temperatures and more redshifted features. This is consistent with the hypothesis that Class C PAHs are hydrocarbon molecules with a high aliphatic/aromatic content ratio found around stars with weak UV radiation fields. The hydrocarbons around CSS 757, KR Cam, and CSS 173 thus represent the composition as condensed in the AGB wind, before entering the interstellar medium where harsh UV radiation alters their chemical structure.

Acknowledgements. The authors wish to thank A. G. G. M. Tielens, J. Bouwman, J. Cami, and P. Degroote for helpful comments and discussion of this study and the general context. K. Smolders, J. Blommaert, L. Decin, and H. Van Winckel acknowledge support from the Fund for Scientific Research of Flanders under the grant G.0470.07. S. Van Eck is an F.N.R.S Research Associate.

\section{References}

Allamandola, L. J., Tielens, A. G. G. M., \& Barker, J. R. 1989, ApJS, 71, 733 Boersma, C., Hony, S., \& Tielens, A. G. G. M. 2006, A\&A, 447, 213

Boersma, C., Bouwman, J., Lahuis, F., et al. 2008, A\&A, 484, 241

Cami, J. 2002, Ph.D. Thesis, University of Amsterdam

Cami, J., Sloan, G. C., Markwick-Kemper, A. J., et al. 2009, ApJ , 690, L122

Cau, P. 2002, A\&A, 392, 203

Cherchneff, I. 2006, A\&A, 456, 1001

Cherchneff, I., Barker, J. R., \& Tielens, A. G. G. M. 1992, ApJ , 401, 269

Chiar, J. E., \& Tielens, A. G. G. M. 2006, ApJ , 637, 774

Cohen, M., Tielens, A. G. G. M., \& Allamandola, L. J. 1985, ApJ , 299, L93

Ferrarotti, A. S., \& Gail, H.-P. 2002, A\&A, 382, 256

Furlan, E., Hartmann, L., Calvet, N., et al. 2006, ApJS, 165, 568

Gielen, C., van Winckel, H., Matsuura, M., et al. 2009, A\&A, 503, 843

Goto, M., Kobayashi, N., Terada, H., et al. 2002, ApJ , 572, 276

Goto, M., Gaessler, W., Hayano, Y., et al. 2003, ApJ , 589, 419

Goto, M., Kwok, S., Takami, H., et al. 2007, ApJ, 662, 389

Gustafsson, B., Edvardsson, B., Eriksson, K., et al. 2008, A\&A, 486, 951

Higdon, S. J. U., Devost, D., Higdon, J. L., et al. 2004, PASP, 116, 975

Hines et al. 2005, FEPS Data Explanatory Supplement, Version 3.0, Pasadena SSC

Hony, S., Heras, A. M., Molster, F. J., et al. 2009, A\&A, 501, 609

Houck, J. R., Roellig, T. L., Van Cleve, J., et al. 2004, in SPIE Conf. Ser. 5487, ed. J. C. Mather, 62

Jäger, C., Mutschke, H., \& Henning, T. 1998, A\&A, 332, 291

Jäger, C., Dorschner, J., Mutschke, H., Posch, T., \& Henning, T. 2003, A\&A, 408, 193

Jura, M., Bohac, C. J., Sargent, B., et al. 2006, ApJ, 637, L45

Keller, L. D., Sloan, G. C., Forrest, W. J., et al. 2008, ApJ , 684, 411

Kraemer, K. E., Sloan, G. C., Bernard-Salas, J., et al. 2006, ApJ , 652, L25

Leger, A., \& Puget, J. L. 1984, A\&A, 137, L5

Lepp, S., \& Dalgarno, A. 1988, ApJ , 324, 553

Li, A., \& Draine, B. T. 2002, ApJ, 572, 232

Mattioda, A. L., Allamandola, L. J., \& Hudgins, D. M. 2005a, ApJ , 629, 1183

Mattioda, A. L., Hudgins, D. M., \& Allamandola, L. J. 2005b, ApJ , 629, 1188

Peeters, E., Hony, S., Van Kerckhoven, C., et al. 2002, A\&A, 390, 1089

Pino, T., Dartois, E., Cao, A., et al. 2008, A\&A, 490, 665

Puget, J. L., \& Leger, A. 1989, ARA\&A, 27, 161

Richer, H. B. 1972, ApJ , 172, L63

Schlegel, D. J., Finkbeiner, D. P., \& Davis, M. 1998, ApJ , 500, 525

Sloan, G. C., Keller, L. D., Forrest, W. J., et al. 2005, ApJ , 632, 956

Sloan, G. C., Jura, M., Duley, W. W., et al. 2007, ApJ , 664, 1144

Sylvester, R. J., Skinner, C. J., \& Barlow, M. J. 1998, MNRAS, 301, 1083

Tielens, A. G. G. M. 2008, ARA\&A, 46, 289

van Diedenhoven, B., Peeters, E., Van Kerckhoven, C., et al. 2004, ApJ , 611, 928

Verhoelst, T., van der Zypen, N., Hony, S., et al. 2009, A\&A, 498, 127

Yang, X. H., Chen, P., Wang, J., et al. 2007, A\&A, 463, 663 